\title{
Community-based molecular and serological surveillance of subclinical malaria in Myanmar
}

Katherine O'Flaherty ${ }^{1,2}$, Win Han Oo ${ }^{3}$, Sophie G. Zaloumis ${ }^{2}$, Julia C. Cutts ${ }^{1,4}$, Kyaw Zayar Aung ${ }^{3}$, Myat Mon Thein ${ }^{3}$, Damien R. Drew ${ }^{1}$, Zahra Razook ${ }^{5}$, Alyssa E. Barry ${ }^{1,4,5}$, Naanki Parischa ${ }^{1}$, Nyi Nyi Zaw ${ }^{3}$, Htin Kyaw Thu ${ }^{3}$, Aung Thi ${ }^{6}$, Wai Yan Min Htay ${ }^{3}$, Aung Paing Soe ${ }^{3}$, Julie A. Simpson ${ }^{2}$, James G. Beeson ${ }^{1,4,7}$, Paul A. Agius ${ }^{1,8,9}$ and Freya J. I. Fowkes ${ }^{1,2,8,10^{*}}$

\begin{abstract}
Background: In the Greater Mekong Subregion (GMS), current malaria surveillance strategies rely on a network of village health volunteers (VHVs) reporting the results of rapid diagnostic tests (RDTs), known to miss many asymptomatic infections. Integration of more sensitive diagnostic molecular and serological measures into the VHV network may improve surveillance of residual malaria transmission in hard-to-reach areas in the region and inform targeted interventions and elimination responses. However, data on residual malaria transmission that would be captured by these measures in the VHV-led testing and treatment surveillance network in the GMS is unknown.

Methods: A total of 114 VHVs were trained to collect dried blood spots from villagers undergoing routine RDTs as part of VHV-led active and passive case detection from April 2015 to June 2016. Samples were subjected to molecular testing (quantitative polymerase chain reaction [aPCR]) to determine Plasmodium falciparum and $P$. vivax infection and serological testing (against $P$. falciparum and $P$. vivax antigens) to determine exposure to $P$. falciparum and P. vivax.

Results: Over 15 months, 114 VHVs performed 32,194 RDTs and collected samples for molecular $(n=13,157)$ and serological $(n=14,128)$ testing. The prevalence of molecular-detectable $P$. falciparum and $P$. vivax infection was $3.2 \%$ compared to the $0.16 \%$ prevalence of Plasmodium spp. by RDT, highlighting the large burden of infections undetected by standard surveillance. Peaks in anti-P. falciparum, but not $P$. vivax, merozoite IgG seroprevalence coincided with seasonal P. falciparum transmission peaks, even in those with no molecularly detectable parasites. At the individual level, antibody seropositivity was associated with reduced odds of contemporaneous $P$. falciparum (OR for PfCSP 0.51 [95\%Cl 0.35, 0.76], $p=0.001$, PfAMA1 0.70 [95\%Cl 0.52, 0.93], $p=0.01$, and PfMSP2 0.81 [95\%Cl $0.61,1.08], p=0.15$ ), but not $P$. vivax infection (OR PvAMA1 $1.02[95 \% \mathrm{Cl} 0.73,1.43], p=0.89$ ) indicating a potential role of immunity in protection against molecular-detectable $P$. falciparum parasitaemia.

(Continued on next page)
\end{abstract}

\footnotetext{
* Correspondence: freya.fowkes@burnet.edu.au

${ }^{1}$ Burnet Institute for Medical Research and Public Health, Melbourne,

Australia

${ }^{2}$ Centre for Epidemiology and Biostatistics, Melbourne School of Population and Global Health, The University of Melbourne, Melbourne, Australia Full list of author information is available at the end of the article
}

C C The Author(s). 2021 Open Access This article is licensed under a Creative Commons Attribution 4.0 International License, which permits use, sharing, adaptation, distribution and reproduction in any medium or format, as long as you give appropriate credit to the original author(s) and the source, provide a link to the Creative Commons licence, and indicate if changes were made. The images or other third party material in this article are included in the article's Creative Commons licence, unless indicated otherwise in a credit line to the material. If material is not included in the article's Creative Commons licence and your intended use is not permitted by statutory regulation or exceeds the permitted use, you will need to obtain permission directly from the copyright holder. To view a copy of this licence, visit http://creativecommons.org/licenses/by/4.0/ The Creative Commons Public Domain Dedication waiver (http://creativecommons.org/publicdomain/zero/1.0/) applies to the data made available in this article, unless otherwise stated in a credit line to the data. 
(Continued from previous page)

Conclusions: We demonstrated that integration and implementation of sample collection for molecular and serological surveillance into networks of VHV servicing hard-to-reach populations in the GMS is feasible, can capture significant levels of ongoing undetected seasonal malaria transmission and has the potential to supplement current routine RDT testing. Improving malaria surveillance by advancing the integration of molecular and serological techniques, through centralised testing approaches or novel point-of-contact tests, will advance progress, and tracking, towards malaria elimination goals in the GMS.

Keywords: Malaria, Plasmodium, Surveillance, Epidemiology, Serosurveillance, Immunity

\section{Background}

The emergence of artemisinin-resistant Plasmodium falciparum within the Greater Mekong Subregion (GMS) has led to the region setting elimination targets for all human malaria by 2030 [1]. In working towards this goal, the incidence of malaria cases and deaths in the GMS fell substantially by $75 \%$ and $93 \%$, respectively, between 2012 and 2017 [2]. Monitoring and surveillance are critical to the elimination of malaria ensuring that progress towards malaria elimination targets can be accurately tracked and ultimately accelerated. However, as the region transitions towards malaria elimination, surveillance becomes increasingly difficult because malaria becomes concentrated in discrete geographical foci, such as border and hard-to-reach areas, and in high-risk populations such as migrant workers and residents of highly forested areas [3-5]. To capture these infections, in many remote areas, surveillance and malaria control strategies are dependent on passive case detection (PCD) and active case detection (ACD) provided by a village health volunteer (VHV) network who administer malaria testing by rapid diagnostic test (RDT) as well as treatment. However, subclinical Plasmodium spp. infections often go undetected because asymptomatic individuals are less likely to seek testing and treatment and, importantly, are generally below the detection limit of conventional RDT diagnostics used in the field. Undetected, and therefore untreated, Plasmodium spp. infections may be an important source of residual malaria transmission [6-10], and failure to detect and eliminate all infections may hinder malaria elimination targets.

Integration of more sensitive diagnostic measures into the VHV network may improve surveillance of residual malaria transmission in hard-to-reach areas in the region. Molecular and serological assays can determine residual malaria transmission not detected by routine diagnostics in the field such as RDT and microscopy. Microscopy is estimated to miss approximately $50 \%$ of infections when compared to molecular methods such as polymerase chain reaction (PCR), and the proportion of missed infections may be greater than $80 \%$ in areas of low transmission (defined at PCR prevalence $<10 \%$ ) [11]. However, the application of sensitive molecular methods such as PCR to detect malaria is mostly utilised as a research tool in many malaria-endemic settings and is yet to be approved and incorporated into routine surveillance in the GMS. Similarly, measuring antibodies specific for malarial antigens is not approved for routine use and may also be a useful surveillance tool to monitoring ongoing malaria transmission in regions approaching malaria elimination as it has the potential to measure both current and recent malaria exposure [12]. Until point-of-contact molecular and serological surveillance tools for malaria become more widely available and approved for use in national malaria control programmes, centralised use of these approaches will be necessary. However, sample collection for surveillance activities may be implemented at the village level by VHV. To date, few studies have been performed in Southeast Asia to investigate the utility of molecular and serological surveillance, and none has been incorporated into the VHV network. Serial cross-sectional research surveys have demonstrated higher blood-stage antimalarial IgG levels and seropositivity amongst those with PCR-detectable subclinical $P$. falciparum and $P$. vivax infection compared to uninfected individuals [13-16], and geospatial analysis has shown that antimalarial antibodies are predictive of ongoing malaria transmission [17]. While these studies suggest the use of serological surveillance may be appropriate for the detection of residual malaria transmission, the feasibility of integrating the approach and the data on residual malaria transmission that would be captured into the PCD/ACD VHV-led testing and treatment surveillance network in the GMS is unknown. To address this knowledge gap, we integrated the collection of participant samples for molecular and serological surveillance into VHV-delivered community-based malaria programmes in Southeast Myanmar to understand the surveillance data that can be captured at this level to inform surveillance of malaria and targeted interventions in elimination settings in the GMS.

\section{Methods}

\section{Study design and sample collection}

Details of the study design and sample collection have previously been reported $[18,19]$ and are detailed in the Supplementary Methodology (Additional File 1). Briefly, 
from April 2015 to June 2016, residents of 114 villages across three south-eastern states/regions (Kayin, Kayah and Bago East) in Myanmar were invited to take part in an open stepped-wedge cluster-randomised control trial designed to estimate the effectiveness of topical insect repellent distributed by VHV on Plasmodium spp. infection (ACTRN12616001434482). This trial was an implementation trial, and the only changes to the malaria programme implementation were the distribution of repellent at a designated month and the addition of dried blood spot sample collection. Like many areas of the GMS, the incidence of malaria cases in Myanmar has reduced substantially in recent years (by $82 \%$ between 2012 and 2017 and by 23\% between 2016 and 2017 alone) [20]. Additionally, large proportions of infections in Myanmar are subclinical and not detected using routine RDT [4, 21-23]. Consistent with routine services provision, VHVs performed RDTs (SD bioline $P f / P v$ combo RDT), treated malaria cases if RDT was positive and collected basic demographic information for the duration of the trial. VHVs aimed to perform a minimum of 20 tests per month, collected by either PCD or ACD, where $\mathrm{PCD}$ refers to villagers presenting to the VHV for testing and ACD refers to VHV seeking villagers for testing (e.g. during health education sessions or household visits). VHV also received 1-day training to perform additional finger prick sample collection. In consenting participants, VHVs collected two drops of blood on Whatman 3-mm filter paper for molecular Plasmodium spp. detection by quantitative PCR (qPCR) and IgG analysis. Filter papers were air-dried, placed in air-tight plastic bags and then stored in specimen collection boxes. At the end of each month, specimen boxes were collected from VHVs by local dioceses malaria officers and stored in refrigerated conditions. Every 2-3 months, samples were transported to Yangon, where they were stored in the refrigerator before shipment to Melbourne, Australia. All participants or parents/guardians provided informed consent, and ethical approval was obtained from the Ethics Review Committee on Medical Research involving Human Subjects, Myanmar Department of Medical Research (21/Ethics/2015), and the Alfred Hospital, Melbourne, Australia (95/15).

\section{Outcome measures}

\section{$P$. falciparum and $P$. vivax infection}

$P$. falciparum or $P$. vivax (or both) infection was determined by SD bioline $P f / P v$ combo RDT according to the manufacturer's instructions and by quantitative PCR (qPCR) as previously described [19] and detailed in Additional File 1 and 2. A positive P. falciparum result included any qPCR-detectable infection where $P$. falciparum was detected, including mixed infections, and likewise for $P$. vivax.

\section{$P$. falciparum and $P$. vivax antigen-specific $\lg G$}

Total IgG in response to $P$. falciparum apical membrane antigen 1 (PfAMA1 [3D7]), merozoite surface protein 2 (PfMSP2 [3D7]) and circumsporozoite (PfCSP [3D7]) and $P$. vivax AMA1 (PvAMA1 [Palo Alto]) was measured by enzyme-linked immunosorbent assay (ELISA) using a robotic liquid handling system (JANUS automated work station, Perkin Elmer), as previously described [24]. Detailed protein expression and ELISA methodology are provided in the Supplementary Methodology (Additional File 1).

\section{Statistical analysis}

Logistic mixed effects modelling was used to estimate the change in IgG seropositivity (seropositive/seronegative) with time (months) and linear mixed effects modelling was used to estimate the change in $\log _{2}$ IgG level (OD 450nm) with time on individual-level observations. The association between $P$. falciparum or $P$. vivax infection detected by qPCR and the following factors were examined using logistic mixed effects modelling: (i) participant IgG level/seropositivity at the time of detection of malaria infection (contemporaneous IgG level/seropositivity) as separate exposures and (ii) participant IgG level/seropositivity at the preceding presentation (lagged IgG level/seropositivity) as independent exposures. Due to a smaller number of $P$. vivax events in the sample with repeated measurements, only contemporaneous IgG level/seropositivity exposures were investigated. Likelihood ratio tests were used to assess whether (i) time as a discrete factor was associated with odds of IgG seropositivity and $\log _{2}$ IgG levels and (ii) an interaction existed between $P$. falciparum or $P$. vivax infection detected by qPCR and time. All mixed effects models included a random effect (intercept) to account for participant repeated measurement, and covariates for time (discrete month), age (years), distribution of topical insect repellent (time-varying monotonic variable as villages transitioned from control to intervention), residential status (village resident/migrant/forest-dwelling resident), sex (male/female) and the operating diocesan areas (Hpa-An and Taungoo Areas in Kayin State, Loikaw Area in Kayah State, Yangon Area in Bago East Region). Models estimating lagged IgG level/seropositivity excluded fixed effects for discrete time (due to model overfit) but included a fixed effect for the time between visits (number of days) for each individual. All data were analysed using Stata14 (StataCorp, Stata Statistical Software: College Station, TX).

\section{Results}

Characteristics of the study population

One hundred and fourteen VHVs (48\% male, median age 26 years (25th and 75th percentiles 21-33) in 102/ 
114 VHV respondents) were trained to undertake malaria diagnosis by RDT and collection of dried blood spot samples in their villages. From April 2015 until June 2016, trained VHVs performed 32,194 RDTs in 114 villages of Southeast Myanmar, as previously described [19]. In a sub-population of consenting participants, VHVs collected 14,938 dried blood spot samples, 810 of which were missing key demographic data $(n=692)$ or were compromised due to improper storage $(n=118)$ and were excluded from all further molecular and serological analyses. The final sub-sample included 14,128 samples from 10,857 individuals (Table 1). In this population, the median age of participants was 20 years (25th and 75th percentiles $10-35$, min and $\max <1-91$ ), and $49.8 \%$ were male $(5402 / 10,857)$. Of all participant samples, $13.1 \%$ were collected from migrants $(1423 / 10,857)$, $39.9 \%$ were collected from village residents $(4329 / 10$, 857 ) and $47 \%$ were collected from forest dwellers $(5105 / 10,857)$ (Table 1). The sub-population that consented to sample collection was broadly reflective of the larger cohort with RDT diagnosis only (Table 1). The majority of participants $(81.3 \%, n=8825 / 10,857)$ provided only one sample throughout the study period (Table S3). There were 2032 participants that contributed multiple samples (total samples $=5303$ ) with a median of two samples per person (25th and 75th percentiles $2-3$, min and max 2-10 samples per person) with a median time between sampling of 118 days (25th and 75th percentile 50-168 days, min and max 4-442 days).

Testing rates and detection of $P$. falciparum and $P$. vivax infection by RDT and qPCR over time

$P$. falciparum and $P$. vivax infection status was determined in all participants by RDT $(n=32,194)$, and 14 , 128 samples were collected for molecular and serological analysis. From this sub-sample, 13,157 samples were suitable for molecular diagnosis by qPCR, and 971 were excluded due to insufficient sample (Table 1). As reported previously [19], the prevalence of Plasmodium spp. infections detected by RDT was $0.16 \%$ (50/32,194; 13 P. falciparum mono-infection [0.04\%], 34 P. vivax mono-infection [0.11\%] and 3 mixed species infections [0.01\%], 29 symptomatic), and in the sub-sample of participants consenting to additional sampling, RDTpositive Plasmodium spp. infection was similarly low at $0.14 \% \quad(20 / 14,128 ; 7 \quad P$. falciparum mono-infection [0.05\%], 12 P. vivax mono-infection [0.08\%] and 1 mixed species infections [0.01\%]) (Table 1 ). In this sub-sample of participants, 13,157 underwent diagnosis by qPCR, and the prevalence of $P$. falciparum and $P$. vivax infection was $3.2 \%(419 / 13,157 ; 207$ P. falciparum mono-

Table 1 Characteristics of VHV performed RDTs and DBS

\begin{tabular}{|c|c|c|c|}
\hline & Total population & Population with RDT only & Population with RDT and DBS \\
\hline Number of participants & $-{ }^{\mathrm{a}}$ & $-{ }^{a}$ & 10,857 \\
\hline Number of RDTs & 32,194 & 18,066 & 14,128 \\
\hline Age (years), median (25th, 75th percentile; min, max) & $18(9,33 ; 0.1,100)$ & $16(8-31,0.1-100)$ & $20(10-35,0.1-91)$ \\
\hline Sex, $\%$ male $(n / N)$ & $50.06(16,116 / 32,194)$ & $50.49(9122 / 18,066)$ & $49.76(5402 / 10,857)$ \\
\hline \multicolumn{4}{|l|}{ Residential status ${ }^{b}, \%(n / N)$} \\
\hline Migrant & $13.71(4415 / 32,191)$ & $15.74(2844 / 18,063)$ & $13.11(1423 / 10,857)$ \\
\hline Village resident & $45.92(14,783 / 32,191)$ & $48.85(8826 / 18,063)$ & $39.87(4329 / 10,857)$ \\
\hline Forest dweller & $40.36(12,993 / 32,191)$ & $35.39(6393 / 18,063)$ & $47.02(5105 / 10,857)$ \\
\hline \multicolumn{4}{|l|}{ RDT, $\%(n / N)$} \\
\hline Negative & $99.84(32,144 / 32,194)$ & $99.83(18,036 / 18,066)$ & $99.86(14,108 / 14,128)$ \\
\hline$P f_{+}$ & $0.04(13 / 32,194)$ & $0.03(6 / 18,066)$ & $0.05(7 / 14,128)$ \\
\hline$P V+$ & $0.11(34 / 32,194)$ & $0.12(22 / 18,066)$ & $0.08(12 / 14,128)$ \\
\hline Mixed & $0.01(3 / 32,194)$ & $0.01(2 / 18,066)$ & $0.01(1 / 14,128)$ \\
\hline \multicolumn{4}{|l|}{$\mathrm{qPCR}^{\mathrm{C}}, \%(n / N)$} \\
\hline Negative & N/A & $\mathrm{N} / \mathrm{A}$ & $96.82(12,738 / 13,157)$ \\
\hline$P f_{+}$ & N/A & $\mathrm{N} / \mathrm{A}$ & $1.57(207 / 13,157)$ \\
\hline$P V+$ & N/A & $\mathrm{N} / \mathrm{A}$ & $0.93(123 / 13,157)$ \\
\hline Mixed & N/A & N/A & $0.68(89 / 13,157)$ \\
\hline
\end{tabular}

$D B S$ dried blood spot, IQR interquartile range, Pf Plasmodium falciparum, PV Plasmodium vivax, qPCR quantitative polymerase chain reaction, $R$ range, $R D T$ rapid diagnostic test, $V H V$ village health volunteer

${ }^{a}$ Individual participant identifiers were only given to consenting participants upon DBS sampling

${ }^{b}$ Residency data missing for 3 participants

' $\mathrm{qPCR}$ detection only performed in participants consenting to DBS collection and N/A for 971 due to insufficient sample 
infection [1.57\%], 123 P. vivax mono-infection [0.93\%] and 89 mixed species infections [0.68\%]), approximately 22-fold higher than by RDT (Table 1).

The average testing rate was 2146 (standard deviation [SD] 554) RDTs per month for the entire VHV network over the course of the study (20 [SD 14] RDTs per village/VHV per month); however, this varied by month, with $2628 \mathrm{RDTs} /$ month in the first month of the study decreasing to 1721 RDTs/month in the last month of the study (Fig. 1a). The average number of dried blood spots collected was 942 (SD 201) samples per month (12 [SD 8] samples per village/VHV per month), with more samples collected per month occurring in the latter part of the study (Fig. 1b). Both qPCR-detectable P. falciparum and $P$. vivax prevalence varied over time (discrete month, likelihood ratio [LR] both $p<0.001$ ) (Fig. 1). $P$. falciparum and $P$. vivax infection was observed to peak in June and July corresponding to the high transmission season in Myanmar (9.67\% and $8.23 \%$ in June and July, respectively) with a second, smaller peak observed in December for P. falciparum (5.93\%, Fig. 1b).

\section{Seroprevalence and levels of antimalarial lgG over time}

To determine the change in the acquired IgG levels and seroprevalence over the study period, IgG responses to $P$. falciparum merozoite and sporozoite antigens PfAMA1, PfMSP2 and PfCSP, and P. vivax merozoite antigen PvAMA1 were determined. Because elution of antibody required less sample than DNA extraction, IgG levels and seroprevalence were determined in all samples collected that had not been compromised and had matched data $(n=14,128)$. The overall seroprevalences for PfAMA1, PfMSP2, PfCSP and PvAMA1 were 38.6 (95\% CI 37.8-39.4), 37.3 (95\% CI 36.5-38.1), 18.7 (95\% CI 18-19.3) and $28.4 \%$ (95\% CI 27.6-29.1), respectively. Both anti-P. falciparum and $P$. vivax IgG level and seroprevalence were associated with time (discrete month, LR all $p<0.001$, Table S4 - S11). Like patterns of qPCR-detectable $P$. falciparum infection, the median levels and overall seroprevalence of anti-PfAMA1 IgG in all observations peaked in June and July of 2015 coinciding with the high transmission season, with an additional peak observed in December of 2015 coinciding with a peak in qPCR detectable $P$. falciparum cases. Peaks in PfMSP2 and PfCSP IgG were only observed to coincide with the second peak in P. falciparum infections in December of 2015 (Fig. 2a, b). Interestingly, these seasonal trends in P. falciparum specific IgG seroprevalence were also reflected in a broader qPCR-negative population (Additional File 2 Figure S4). The median levels and seroprevalence of anti-PvAMA1 IgG fluctuated and increased slightly throughout the study period; however, these changes were largely unreflective of changes in qPCR detected $P$. vivax prevalence (Fig. 2c, d).

\section{Association between IgG and $P$. falciparum and $P$. vivax infection}

To determine the association between IgG seropositivity on $P$. falciparum and $P$. vivax qPCR-detectable infection at the individual level, we performed logistic mixed effects modelling, including all data and fixed effects for age, sex, region, residential status, time and repellent distribution, and random effects for repeated participant sampling. Seropositivity for anti-PfAMA1, PfMSP2 and PfCSP IgG was associated with a reduction in the odds of contemporaneous qPCR-detectable $P$. falciparum infection by between 19 and 49\%; however, a wide confidence interval was observed in the PfMSP2 estimate (anti-PfAMA1 OR 0.70 (95\%CI 0.52, 0.93), $p=0.01$, anti-PfMSP2 OR 0.81 (95\%CI 0.61, 1.08), $p=0.15$, antiPfCSP OR 0.51 (95\%CI 0.35, 0.76), $p=0.001$; Table 2). Similarly, a twofold increase in the levels of anti-PfMSP2 and $P f C S P$ were associated with a 12 and $10 \%$ reduction, respectively, in the odds of $P$. falciparum infection (PfMSP2 OR 0.88 (95\%CI 0.80, 0.96), $p=0.005$, antiPfCSP OR 0.90 (95\%CI 0.83, 0.98), $p=0.02$, Table 2). Increased anti-PfAMA1 IgG level was not associated with the odds of qPCR-detectable $P$. falciparum infection (OR 0.94 (95\%CI 0.84, 1.06), $p=0.34$, Table 2). When lagged, IgG level/seroprevalence was examined as the exposure (i.e. using the preceding IgG measurement), in participants with repeated measurements after including a fixed effect for the time between sampling (days) anti-P. falciparum IgG level/seropositivity was not associated with qPCR-detectable P. falciparum (Table 2). Anti-PvAMA1 IgG level and seropositivity were not associated with contemporaneous qPCR-detectable $P$. vivax infection (OR 1.02 (95\%CI $0.73,1.43), p=0.89$ and OR 0.98 (95\%CI 0.86, 1.12), $p=0.75$, Table 2, respectively). Due to a smaller number of $P$. vivax-positive participants with repeated measurements $(n=68)$, lagged effects of IgG were not estimated.

\section{Discussion}

VHVs are the cornerstone of malaria control and surveillance activities in many malaria-endemic settings. By training VHV delivering routine malaria services to integrate sample collection for molecular and serological surveillance in their village, we demonstrated that surveillance using highly sensitive molecular methods in samples collected by VHV is feasible and can detect a significant subclinical reservoir of infection undetected by standard surveillance using RDTs. Furthermore, serological data reflected monthly trends in $P$. falciparum and $P$. vivax infection, even in those with no detectable parasites, indicating that this surveillance approach can capture significant levels of ongoing undetected seasonal malaria transmission. Integration of molecular and serological surveillance into the extensive VHV network may 

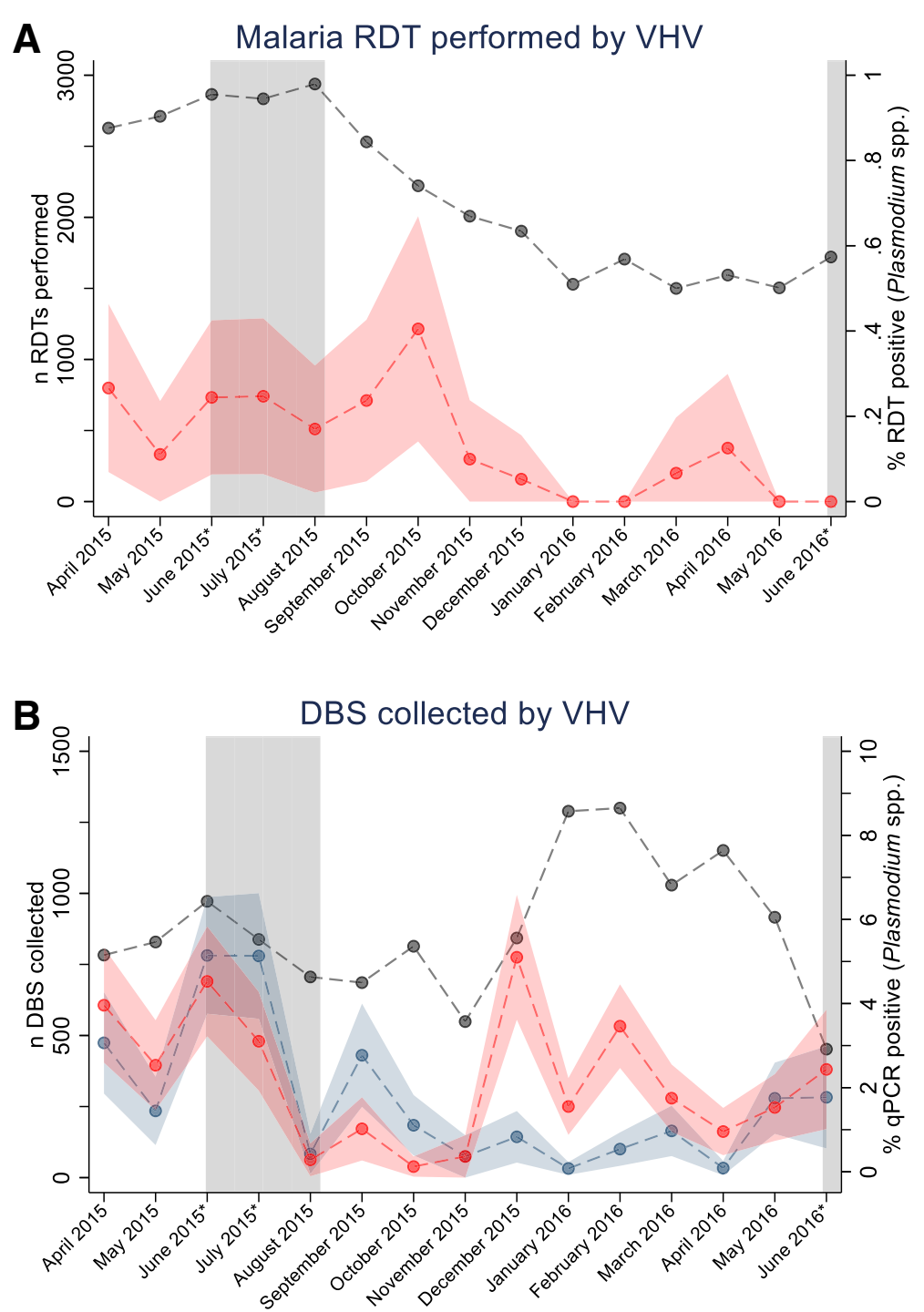

Fig. 1 Number of RDTs collected by VHV (grey) and prevalence of Plasmodium spp. infection by RDT (95\% Cl [red]) (a) and number of DBS collected by VHV (grey) and prevalence of Plasmodium spp. infection (95\% Cl) by qPCR (P. falciparum [red] and P. vivax [blue]) (b) by month over the study period. The high transmission season is shown in grey and months marked with an asterisk. Lines connecting data points are intended only to highlight patterns and not to suggest a continuum between data points

be an effective tool for monitoring residual malaria transmission in hard-to-reach pre-elimination settings by supplementing an established VHV surveillance network currently dependant on RDTs. Further, the addition of this kind of supplemented surveillance could support centralised malaria control strategies such as resource allocation until more suitable field-deployable tests are available to provide VHV with real-time treatment decision-making tools. Improving malaria surveillance will advance progress, and tracking, towards malaria elimination goals in the GMS.

RDT diagnosis and reporting of malaria cases by VHVs is the key indicator for progressing towards malaria elimination goals in the GMS. Like in much of the region, a large proportion of Plasmodium spp. infections found in participating villages were missed by RDT diagnosis with $\sim 22$-fold greater prevalence of qPCRdetectable infections over the entire study period. There was significant variation in qPCR-detectable infection over time with peaks of Plasmodium spp. infection in the rainy season, where malaria transmission increases, which were not detected by RDT, nor subsequently treated. There is increasing evidence that these untreated molecular-detected infections have the potential to contribute to ongoing malaria transmission, and despite not being transmitted as effectively to mosquitoes as microscopically detected infections, the greater prevalence of subclinical compared to clinical infections in 


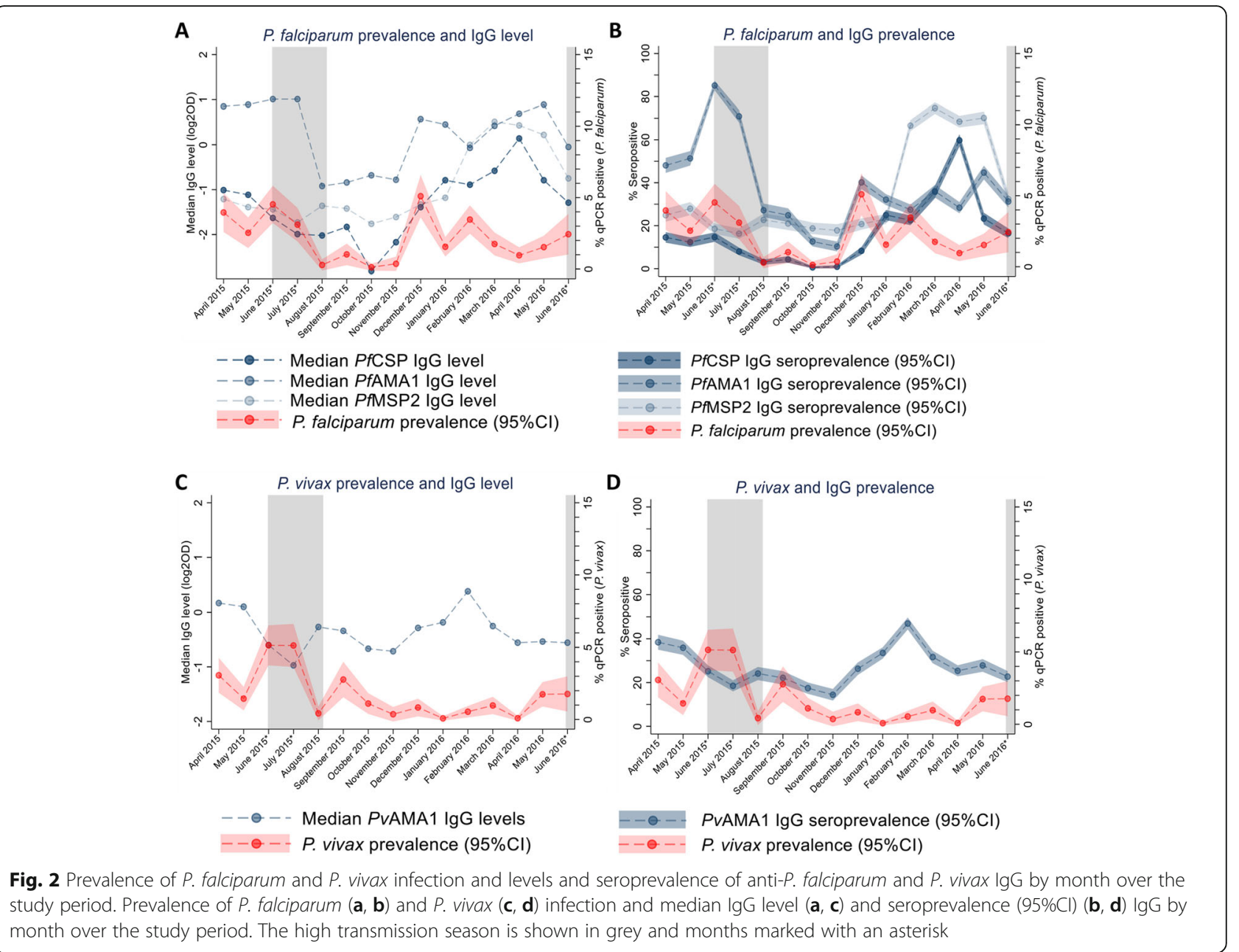

Table 2 Association between qPCR-detectable $P$. falciparum or $P$. vivax infection and antibody response

\begin{tabular}{|c|c|c|c|c|c|c|}
\hline & \multicolumn{3}{|c|}{ Contemporaneous infection } & \multicolumn{3}{|c|}{ Infection at next presentation } \\
\hline & $\mathrm{aOR}^{\mathrm{a}}$ & $(95 \% \mathrm{Cl})$ & $p$ value & $\mathrm{aOR}^{\mathrm{b}}$ & $(95 \% \mathrm{Cl})$ & $p$ value \\
\hline \multicolumn{7}{|l|}{ PfAMA1 lgG } \\
\hline Seropositive & 0.70 & $(0.52,0.93)$ & 0.01 & 0.98 & $(0.53,1.80)$ & 0.95 \\
\hline Level $\left(\log _{2} \mathrm{OD} 450 \mathrm{~nm}\right)$ & 0.94 & $(0.84,1.06)$ & 0.34 & 0.91 & $(0.73,1.10)$ & 0.33 \\
\hline \multicolumn{7}{|l|}{ PAMSP2 lgG } \\
\hline Seropositive & 0.81 & $(0.61,1.08)$ & 0.15 & 1.27 & $(0.70,2.29)$ & 0.43 \\
\hline Level $\left(\log _{2} \mathrm{OD} 450 \mathrm{~nm}\right)$ & 0.88 & $(0.80,0.96)$ & 0.005 & 0.92 & $(0.77,1.11)$ & 0.40 \\
\hline \multicolumn{7}{|l|}{ PfCSP IgG } \\
\hline Seropositive & 0.51 & $(0.35,0.76)$ & 0.001 & 0.29 & $(0.07,1.21)$ & 0.09 \\
\hline Level $\left(\log _{2} \mathrm{OD} 450 \mathrm{~nm}\right)$ & 0.90 & $(0.83,0.98)$ & 0.02 & 1.02 & $(0.85,1.24)$ & 0.81 \\
\hline \multicolumn{7}{|l|}{ PVAMA1 $\lg G^{c}$} \\
\hline Seropositive & 1.02 & $(0.73,1.43)$ & 0.89 & - & & \\
\hline Level $\left(\log _{2} \mathrm{OD} 450 \mathrm{~nm}\right)$ & 0.98 & $(0.86,1.12)$ & 0.75 & - & & \\
\hline
\end{tabular}

Adjusted for ${ }^{a}$ age (years), sex, region, residential status, time (discrete month) and repellent distribution or ${ }^{b}$ age (years), sex, region, residential status, time since the last measurement (days) and repellent distribution; ' only contemporaneous lgG level/seropositivity exposures were estimated for $P$. vivax infections due to a small number of events for individuals with measurements performed on at least two occasions. Estimates for antigen- specific lgG seropositivity or level are derived from separate equations, (Additional File 2 Tables S3-S10) $a O R$ adjusted odds ratio, $95 \% \mathrm{Cl} 95 \%$ confidence interval 
low-transmission settings is cause for concern [25]. Detection of these infections is becoming increasingly difficult because in the elimination phase, malaria is concentrated in hard-to-reach areas and high-risk populations. Therefore, additional surveillance tools deployed at the VHV level may help target interventions aimed at reducing the subclinical burden of malaria and advance the malaria elimination agenda in this region.

Serosurveillance does not provide a diagnosis of a current Plasmodium spp. infection. Rather, at a population level, antimalarial antibodies may indicate recent Plasmodium spp. transmission and exposure and therefore represent a useful tool for malaria microstratification to inform resource allocation and targeted implementation of malaria control and elimination interventions by NMCPs if integrated into national surveillance. The observed peaks in anti- $P$. falciparum merozoite IgG seroprevalence coinciding approximately with peaks of PCR-detectable $P$. falciparum infection, even in parasite negative individuals, indicate that malarial serology captured ongoing malaria transmission in the population not detected by routine RDTs. However, the magnitude of antibody seroprevalence was significantly greater than the prevalence of PCR detectable infections. Caution must be exercised in estimating the prevalence of circulating infections based on serology, which may be overestimated particularly in regions where the malaria burden is transitioning from malaria control to elimination phases, as antibodies can persist for extended periods [26, 27]. This study was conducted amid Myanmar's transition from malaria control to elimination and following large-scale reductions in Plasmodium spp. transmission in the study region [28]. The higher seroprevalence compared to PCR prevalence of $P$. falciparum and $P$. vivax infections may reflect cumulative incidence in the region over this time period [28, 29]. Between 2005 and 2014, immediately prior to the commencement of this study in 2015, the prevalence of malaria detected by RDT declined in Kayah, Kayin and Bago states by more than $74 \%$ (from 3.2 to $1.7 \%, 0.86$ to $0.52 \%$, and 0.44 to $0.07 \%$, respectively) [29]. The historical prevalence in the 10 years prior to this study are considerably higher than the $0.16 \%$ RDT positivity reported here and may result in the cumulative incidence of antimalarial antibody responses observed because the mean antimalarial antibody half-lives of the antigens included in this study (selected because their immunogenicity and half-lives are relatively well-characterised in the GMS) may range between 6 months and 7 years IgG $[27,30,31]$. As such, serosurveillance may be a more informative tool as antibodies wane in the broader population and is limited to only exposed populations in elimination phases or alternatively different sets of antigens could be defined which can quantitatively capture very recent molecular infection events [14]. This study was not designed to identify serological biomarkers of recent exposure to molecularly detectable infections as this would require detailed longitudinal follow-up and accurate participant exposure history. Several recent studies have demonstrated the utility in combining novel combinations of antigens to predict recent Plasmodium spp. infection exposure detected by microscopy, clinical incidence and relapse with $P$. vivax, across diverse malaria-endemic populations [14, 32-34]. However, the efforts to identify reliable, quantitative antibody signatures of recent exposure to malaria parasites are ongoing $[35,36]$, and there is currently a lack of consensus on antigen-specific antibody responses which discriminate against recent and historical exposures, in particular, of molecularly detectable infections. We investigated $P$. falciparum antigens based on the 3D7 allelic variant. There is limited data on antigen allelic diversity in Myanmar, but previous studies have shown that the majority of circulating MSP alleles are of the 3D7 type [37] and that variation in IgG response to the different allelic variants of PfMSP2 across multiple sites in Myanmar and the Greater Mekong Subregion is minimal [38]. Furthermore, antibody responses specific for epitopes within conserved domains of merozoite antigens have been shown to illicit strain transcending antibody responses [39], so inclusion of additional antigenic variants may not have impacted our study conclusions. However, further refinement of choice and number of antigens and allelic types across different areas of Myanmar and the GMS is warranted to inform the utility of serological findings in the region. Regardless of ultimate antigen selection or malaria burden phase, any new tools need to be validated with respect to the health system platform, such as the VHV-led delivery of malaria services, which will ultimately be integrated into.

In individual-level analyses, we observed a reduction in the odds of concurrent qPCR detectable P. falciparum infection in participants seropositive for $P$. falciparumspecific IgG. Recent longitudinal data from the GMS has shown that within infected individuals, parasite density oscillates frequently and that spontaneous clearance of PCR-detectable P. falciparum infection (i.e. in the absence of treatment) occurs regularly with the median time to resolution of infection of around 2 months for $P$. falciparum and 6 months for P. vivax [40]. We hypothesise that boosting of antibodies upon exposure to parasites may then contribute to clearance of subclinical parasitaemia which may explain the observed reduction in the odds of a contemporaneous qPCR-detectable $P$. falciparum infection in seropositive individuals when sampled in the VHV network. This study provides evidence of potential protective immunity against molecular-detectable infections, which to date has only 
been established to reduce clinical malaria and high parasitaemia in numerous high-transmission populations [41]. We were unable to show protection against prospective infection; however, there were fewer repeated measures (returning participants were less than $20 \%$ of all samples) with large variation in the time between tests. Further studies investigating the response to and role of anti-malarial antibodies in the persistence of molecular-detectable subclinical infections would be valuable to inform the utility and sampling framework of serological surveillance in areas where the majority of the malaria burden remains undetected by conventional surveillance.

The aim of this study was to investigate malaria incidence and serological data within an existing national malaria surveillance strategy, in this case, the VHV network. In the national VHV testing strategy, some individuals will be sampled multiple times and others only once, that is, malaria incidence is representative of the population that is sampled by VHV which may not be truly representative of the general population per se. Repeated sampling of a subset of individuals within the same population risks overestimating malaria incidence when the same individuals present multiple times with a single infection. In this study, which accounted for repeated measures in statistical analyses, only one participant contributing multiple samples was $\mathrm{qPCR}$ positive more than once, in samples collected 10 months apart. Given the estimated clearance time of sub-clinical P. falciparum infection in the region is estimated to be under 3 months [40], these likely represent two separate infection events. Therefore, in the present study, multiple samples from individual participants would not have biassed estimates of malaria incidence in samples collected by VHV. Nonetheless, accurate estimates of malaria incidence due to repeated sampling of a subset of individuals should be considered in the broader national malaria surveillance strategy, given that the sampling strategy is the same as current RDT testing implemented nationally at the VHV level.

Sampling at the VHV level may facilitate increased surveillance in rural and hard-to-reach areas. In this study, VHVs aimed to collect a minimum of 20 RDTs each month in accordance with programme implementation through PCD/ACD; however, data could not be stratified based on the VHV case detection method as this data was not collected. As such, we are unable to determine whether PCD alone would be sufficient to accurately capture the subclinical malaria burden or changes in serological markers reported here. The total number of RDTs declined across the study period, and this is most likely due to the declining motivation of the VHV to undertake RDTs which has been described in the region in areas approaching elimination where positive RDT results are infrequent [42]. This was not reflected in sample collection, the total number of which fluctuated across the study. Participation in sample collection, however, occurred in less than half of participants receiving testing by RDT which, from anecdotal reports, may have been due to reluctance to commit further time to complete the additional informed consent and testing procedures (in Myanmar, dried blood spot sampling is not part of routine VHV services and considered research; therefore, additional consent is required). However, participants contributing samples were broadly similar to those contributing RDTs. Until dried blood spot sampling is considered part of routine sample collection, routinely collected RDTs could be utilised as an alternative source of sample for molecular and serological surveillance of malaria to overcome participation issues [43-45], but their utility for molecular assays, given low parasite densities, needs to be investigated in the Myanmar context. Importantly, capacity building for local laboratories needs to be prioritised as well as understanding how this data will operationally be integrated into the national surveillance electronic system in order to inform programmatic decisions.

\section{Conclusions}

Current malaria surveillance strategies in the GMS rely on VHV reporting the results of insensitive RDTs that do not detect low-density infections. These undetected, untreated infections may contribute to ongoing malaria transmission and have the potential to hinder progress towards malaria elimination. We have shown that sample collection for molecular and serological surveillance can be integrated and implemented in communitydelivered VHV health programmes in hard-to-reach populations and has the potential to capture ongoing malaria transmission at the individual and population levels. While molecular and serological testing in this study was undertaken after fieldwork was completed, away from the villages, data captured by sampling at the VHV level has the potential to inform programmatic decisions such as resource deployment by national malaria control programmes. With the increasing development and availability of easy-to-use, portable point-of-contact molecular and serological tests, there is future potential for VHV to undertake molecular and serological malaria surveillance and report detected cases in near real time. This will not only advance current routine VHV-led malaria surveillance, particularly in hard-to-reach areas, but will also reinforce ownership of malaria surveillance and the malaria elimination agenda to the community who will ultimately play a key role in reaching malaria elimination goals in the GMS. 


\section{Abbreviations}

ACD: Active case detection; AMA1: Apical membrane antigen 1; CSP: Circumsporozoite protein; ELISA: Enzyme-linked immunosorbent assay; GMS: Greater Mekong Subregion; Ig: Immunoglobulin; LR: Likelihood ratio; MSP2: Merozoite surface protein 2; OR: Odds ratio; PCD: Passive case detection; PCR: Polymerase chain reaction; Pf: Plasmodium falciparum; PV: P. vivax; qPCR: Quantitative PCR; RDT: Rapid diagnostic test; SD: Standard deviation; VHV: Village health volunteer

\section{Supplementary Information}

The online version contains supplementary material available at https://doi. org/10.1186/s12916-021-01993-8.

Additional file 1. Supplementary methodology.

Additional file 2. Supplementary tables and figures.

\section{Acknowledgements}

We would like to thank the local communities and VHVs for their participation in the research; KMSS staff Win Tun Kyi, Augustine Tual Sian Piang, Min Thant Zin Latt, Nwe Ni Aye and Khine Zar Lwin (KMSS National Office, Yangon, Myanmar); Joseph Maung Win, Tun Tun Aung, Richard Joseph, Shein Thu Ag, Thet Naing and Myo Tint (KMSS Yangon, Yangon, Myanmar); Ludovico Saw Piko, Benedetta, Win Win Aye, Ngo Petru, Maurice Nyo, Daniel Win and Saw Golbert (KMSS Taunggo, Taunggo, Myanmar); Albino Htwe Win, Tin Aung, Perpetua Aye Aye Mon, Mupaula, John Bosco, Alfred, John Min Aung and Poe Rah (KMSS Loikaw, Loikaw, Myanmar); Paul Thar San, Saw Isidore, Theresa, Saw Micheal and Saw Pho Muo (KMSS Hpa An, Hpa An, Myanmar) for the local advocacy, coordination and preliminary planning of field work; Burnet Institute staff Toe Than Tun, Poe Poe Aung, Phone Myint Win, Poe Poe Aung, Nyi Nyi Zaw, Htin Kyaw Thu, Ai Pao Yawn, Lia Burns, Naanki Pasricha, Ricardo Ataíde and Nicole Romero for contributing towards technical, coordination and management support and contextual inputs; Alissa Robertson and Josh Charles for the assistance in sample processing; and Myanmar Ministry of Health and Sports staff Dr. Thandar Lwin, Dr. Thaung Hlaing, Dr. Than Naing Soe and Dr. Kyawt Mon Win for the technical and administrative support.

\section{Authors' contributions}

FJIF conceived the study. PAA, JCC, WHO and FJIF designed the study. KO, $\mathrm{ZR}, \mathrm{AEB}, \mathrm{DRD}$ and JGB generated and interpreted the laboratory data. $\mathrm{KO}$, SGZ, JAS and FJIF performed and interpreted the data analyses. KO and FJIF wrote the first draft of the manuscript. All authors contributed to the writing of the manuscript and approved the final submitted version and agreed to publication.

\section{Funding}

The implementation of malaria services in Myanmar was supported by the Three Millennium Development Goal (3MDG) fund (BI-MARC-3MDG-C2-1400089847). Further funding was received from the Australian Research Council (Future Fellowship to FJIF, FT130101122) and the National Health and Medical Research Council (Australian Centre for Research Excellence in Malaria Elimination (ACREME) to FJIF, JAS, JGB (1134989); Career Development Fellowship to FJIF (1166753); Senior Research Fellowship to JAS (1104975); Investigator Grant (1173046) and Programme Grant (1092789) to JGB; and its Independent Research Institute Infrastructure Support Scheme). The authors gratefully acknowledge the contribution to this work of the Victorian Operational Infrastructure Support Program received by the Burnet Institute. The funders had no role in the study design, data collection and analysis, decision to publish or preparation of the manuscript.

\section{Availability of data and materials}

Data cannot be made publicly available because it would breach compliance with the ethical framework of the Ethics Review Committee on Medical Research Involving Human Subjects, Department of Medical Research, Myanmar Ministry of Health and Sports. De-identified individual participant data will be available after publication from the data custodian(s) to applicants who provide a sound proposal to the Ethics Review Committee on Medical Research Involving Human Subjects, Department of Medical Research, Myanmar Ministry of Health and Sports (No. 5 Ziwaka Road, Dagon
PO Yangon, Myanmar; (+95) 01375447 extension 118; moc.liamg@5102rmdcre) contingent of their approval.

\section{Declarations}

\section{Ethics approval and consent to participate}

The primary trial is registered in the Australian New Zealand Clinical Trials Registry (ACTRN12616001434482; approved retrospectively 14 October 2016) and was approved by the Ethics Review Committee on Medical Research involving Human Subjects, Department of Medical Research, Ministry of Health and Sports, Myanmar Government (\#21/Ethics/2015; extended approval \#Ethics/DMR/2016/020), and the Alfred Hospital, Melbourne, Australia (95/15). The ethics review committee of the Department of Medical Research, Ministry of Health and Sports, requested that there be no commercial advantage for the product during the trial. Consequently, the repellent was provided in plain unbranded tubes, and the investigators registered the trial after completion of fieldwork (but prior to the commencement of data analysis) to minimise public disclosure. The study protocol has been published previously [18], and the study is reported according to the CONSORT guidelines of reporting a stepped-wedge cluster randomised trial [19].

\section{Competing interests}

The authors declare that they have no competing interests.

\section{Author details}

${ }^{1}$ Burnet Institute for Medical Research and Public Health, Melbourne, Australia. ${ }^{2}$ Centre for Epidemiology and Biostatistics, Melbourne School of Population and Global Health, The University of Melbourne, Melbourne, Australia. ${ }^{3}$ Burnet Institute Myanmar, Yangon, Myanmar. ${ }^{4}$ Department of Medicine, University of Melbourne, Melbourne, Australia. ${ }^{5}$ School of Medicine, Deakin University, Geelong, Australia. ${ }^{6}$ Department of Public Health, Myanmar Ministry of Health, Nay Pyi Taw, Myanmar. ${ }^{7}$ Department of Microbiology and Central Clinical School, Monash University, Melbourne, Australia. ${ }^{8}$ Department of Epidemiology and Preventative Medicine, Monash University, Melbourne, Australia. ${ }^{9}$ Judith Lumley Centre, La Trobe University, Melbourne, Australia. ${ }^{10}$ Department of Infectious Diseases, Monash University, Melbourne, Australia.

Received: 19 November 2020 Accepted: 27 April 2021

Published online: 28 May 2021

\section{References}

1. WHO. Eliminating malaria in the Greater Mekong Subregion. Geneva: World Health Organization; 2016.

2. WHO. World Malaria Report 2018. Geneva: World Health Organization; 2018

3. Cotter C, Sturrock HJ, Hsiang MS, Liu J, Phillips AA, Hwang J, et al. The changing epidemiology of malaria elimination: new strategies for new challenges. Lancet. 2013;382(9895):900-11. https://doi.org/10.1016/S01406736(13)60310-4.

4. Zaw MT, Thant M, Hlaing TM, Aung NZ, Thu M, Phumchuea K, et al. Asymptomatic and sub-microscopic malaria infection in Kayah State, eastern Myanmar. Malar J. 2017;16(1):138. https://doi.org/10.1186/s12936-017-1789-9.

5. Edwards HM, Canavati SE, Rang C, Ly P, Sovannaroth S, Canier L, et al. Novel cross-border approaches to optimise identification of asymptomatic and artemisinin-resistant Plasmodium infection in mobile populations crossing Cambodian borders. PloS one. 2015;10(9):e0124300. https://doi.org/10.1371/ journal.pone.0124300.

6. Schneider P, Bousema JT, Gouagna LC, Otieno S, van de Vegte-Bolmer M, Omar SA, et al. Submicroscopic Plasmodium falciparum gametocyte densities frequently result in mosquito infection. Am J Trop Med Hyg. 2007; 76(3):470-4. https://doi.org/10.4269/ajtmh.2007.76.470.

7. Coleman RE, Kumpitak C, Ponlawat A, Maneechai N, Phunkitchar V, Rachapaew N, et al. Infectivity of asymptomatic Plasmodium-infected human populations to Anopheles dirus mosquitoes in western Thailand. J Med Entomol. 2004;41 (2):201-8. https://doi.org/10.1603/0022-2585-41.2.201.

8. Ouedraogo AL, Bousema T, Schneider P, de Vlas SJ, Ilboudo-Sanogo E, Cuzin-Ouattara N, et al. Substantial contribution of submicroscopical Plasmodium falciparum gametocyte carriage to the infectious reservoir in an area of seasonal transmission. PloS one. 2009:4(12):e8410. https://doi. org/10.1371/journal.pone.0008410. 
9. Okell LC, Bousema T, Griffin JT, Ouedraogo AL, Ghani AC, Drakeley CJ. Factors determining the occurrence of submicroscopic malaria infections and their relevance for control. Nat Commun. 2012;3(1):1237. https://doi. org/10.1038/ncomms2241.

10. Chaumeau V, Kajeechiwa L, Fustec B, Landier J, Naw Nyo S, Nay Hsel S, et al. Contribution of asymptomatic Plasmodium infections to the transmission of malaria in Kayin State, Myanmar. J Infect Dis. 2019;219(9):1499-1509.

11. Okell LC, Ghani AC, Lyons E, Drakeley CJ. Submicroscopic infection in Plasmodium falciparum-endemic populations: a systematic review and meta-analysis. J Infect Dis. 2009;200(10):1509-17. https://doi.org/10.1086/ 644781

12. Elliott SR, Fowkes FJ, Richards JS, Reiling L, Drew DR, Beeson JG. Research priorities for the development and implementation of serological tools for malaria surveillance. F1000prime Reports. 2014;6:100.

13. Kerkhof K, Sluydts V, Willen L, Kim S, Canier L, Heng S, et al. Serological markers to measure recent changes in malaria at population level in Cambodia. Malar J. 2016;15(1):529. https://doi.org/10.1186/s12936-016-1576-z.

14. Longley RJ, White MT, Takashima E, Brewster J, Morita M, Harbers M, et al. Development and validation of serological markers for detecting recent Plasmodium vivax infection. Nat Med. 2020;26(5):741-9. https://doi.org/10.1 038/541591-020-0841-4.

15. Ya-Umphan P, Cerqueira D, Cottrell G, Parker DM, Fowkes FJl, Nosten F, et al. Anopheles salivary biomarker as a proxy for estimating Plasmodium falciparum malaria exposure on the Thailand-Myanmar border. Am J Trop Med Hyg. 2018;99(2):350-6. https://doi.org/10.4269/ajtmh.18-0081.

16. Baum E, Sattabongkot J, Sirichaisinthop J, Kiattibutr K, Jain A, Taghavian O, et al. Common asymptomatic and submicroscopic malaria infections in Western Thailand revealed in longitudinal molecular and serological studies: a challenge to malaria elimination. Malar J. 2016;15(1):333. https://doi.org/1 0.1186/s12936-016-1393-4

17. Kerkhof K, Sluydts V, Heng S, Kim S, Pareyn M, Willen L, et al. Geographical patterns of malaria transmission based on serological markers for falciparum and vivax malaria in Ratanakiri, Cambodia. Malar J. 2016;15(1):510. https:// doi.org/10.1186/s12936-016-1558-1.

18. Win Han O, Cutts JC, Agius PA, Kyaw Zayar A, Poe Poe A, Aung T, et al. Effectiveness of repellent delivered through village health volunteers on malaria incidence in villages in South-East Myanmar: a stepped-wedge cluster-randomised controlled trial protocol. BMC Infect Dis. 2018;18(1):663. https://doi.org/10.1186/s12879-018-3566-y.

19. Agius PA, Cutts JC, Han Oo W, Thi A, O'Flaherty K, Zayar Aung K, et al. Evaluation of the effectiveness of topical repellent distributed by village health volunteer networks against Plasmodium spp. infection in Myanmar: a stepped-wedge cluster randomised trial. PLoS Med. 2020;17(8):e1003177.

20. WHO. The Mekong Malaria Elimination Programme: countries of the Greater Mekong are stepping up to end malaria. Geneva: World Health Organization; 2018.

21. Imwong M, Hanchana S, Malleret B, Rénia L, Day NPJ, Dondorp A, et al. High-throughput ultrasensitive molecular techniques for quantifying lowdensity malaria parasitemias. Journal of Clinical Microbiology. 2014;52(9): 3303-9. https://doi.org/10.1128/JCM.01057-14.

22. Huang F, Takala-Harrison S, Liu H, Xu JW, Yang HL, Adams M, et al. Prevalence of clinical and subclinical Plasmodium falciparum and Plasmodium vivax malaria in two remote rural communities on the Myanmar-China border. Am J Trop Med Hyg. 2017;97(5):1524-31. https:// doi.org/10.4269/ajtmh.17-0167.

23. Ghinai I, Cook J, Hla TT, Htet HM, Hall T, Lubis IN, et al. Malaria epidemiology in central Myanmar: identification of a multi-species asymptomatic reservoir of infection. Malar J. 2017;16(1):16. https://doi.org/1 0.1186/s12936-016-1651-5.

24. Charnaud SC, McGready R, Herten-Crabb A, Powell R, Guy A, Langer C, et al. Maternal-foetal transfer of Plasmodium falciparum and Plasmodium vivax antibodies in a low transmission setting. Scientific Reports. 2016;6(1):20859. https://doi.org/10.1038/srep20859.

25. Slater HC, Ross A, Felger I, Hofmann NE, Robinson L, Cook J, et al. The temporal dynamics and infectiousness of subpatent Plasmodium falciparum infections in relation to parasite density. Nat Commun. 2019;10(1):1433. https://doi.org/10.1038/s41467-019-09441-1.

26. Mugyenyi CK, Elliott SR, Yap XZ, Feng G, Boeuf P, Fegan G, et al. Declining malaria transmission differentially impacts the maintenance of humoral immunity to Plasmodium falciparum in children. J Infect Dis. 2017;216(7): 887-98. https://doi.org/10.1093/infdis/jix370.
27. Fowkes FJ, McGready R, Cross NJ, Hommel M, Simpson JA, Elliott SR, et al. New insights into acquisition, boosting, and longevity of immunity to malaria in pregnant women. J Infect Dis. 2012;206(10):1612-21. https://doi. org/10.1093/infdis/jis566.

28. DoPHMoHa S. The Republic of the Union of Myanamar: national strategic plan 2016-2020 for intensifying malaria control and accelerating progress towards malaria elimination; 2017.

29. Mu TT, Sein AA, Kyi TT, Min M, Aung NM, Anstey NM, et al. Malaria incidence in Myanmar 2005-2014: steady but fragile progress towards elimination. Malar J. 2016;15(1):503. https://doi.org/10.1186/s12936-016-1 567-0.

30. Wipasa J, Suphavilai C, Okell LC, Cook J, Corran PH, Thaikla K, et al. Longlived antibody and B cell memory responses to the human malaria parasites, Plasmodium falciparum and Plasmodium vivax. PLoS Pathogens. 2010;6(2):e1000770. https://doi.org/10.1371/journal.ppat.1000770.

31. Longley RJ, Sattabongkot J, Mueller I. Insights into the naturally acquired immune response to Plasmodium vivax malaria. Parasitol. 2016;143(2):15470. https://doi.org/10.1017/S0031182015000670.

32. Helb DA, Tetteh KK, Felgner PL, Skinner J, Hubbard A, Arinaitwe E, et al. Novel serologic biomarkers provide accurate estimates of recent Plasmodium falciparum exposure for individuals and communities. Proc Natl Acad Sci U S A. 2015;112(32):E4438-47. https://doi.org/10.1073/pna S.1501705112.

33. Surendra H, Supargiyono, Ahmad RA, Kusumasari RA, Rahayujati TB, Damayanti SY, et al. Using health facility-based serological surveillance to predict receptive areas at risk of malaria outbreaks in elimination areas. BMC Med. 2020;18(1):9

34. Ondigo BN, Hamre KES, Frosch AEP, Ayodo G, White MT, John CC. Antibody Profiles to P. falciparum antigens over time characterize acute and longterm malaria exposure in an area of low and unstable transmission. Am J Trop Med Hyg. 2020;103(6):2189-97. https://doi.org/10.4269/ajtmh.19-0480.

35. Yman V, White MT, Asghar M, Sundling C, Sondén K, Draper SJ, et al. Antibody responses to merozoite antigens after natural Plasmodium falciparum infection: kinetics and longevity in absence of re-exposure. BMC medicine. 2019;17(1):22. https://doi.org/10.1186/s12916-019-1255-3.

36. Greenhouse B, Daily J, Guinovart C, Goncalves B, Beeson J, Bell D, et al. Priority use cases for antibody-detecting assays of recent malaria exposure as tools to achieve and sustain malaria elimination. Gates open research. 2019;3:131. https://doi.org/10.12688/gatesopenres.12897.1.

37. Lê HG, Kang JM, Jun H, Lee J, Thái TL, Myint MK, et al. Changing pattern of the genetic diversities of Plasmodium falciparum merozoite surface protein1 and merozoite surface protein-2 in Myanmar isolates. Malar J. 2019;18(1): 241. https://doi.org/10.1186/s12936-019-2879-7.

38. Ataide R, Ashley EA, Powell R, Chan JA, Malloy MJ, O'Flaherty K, et al. Host immunity to Plasmodium falciparum and the assessment of emerging artemisinin resistance in a multinational cohort. Proc Natl Acad Sci U S A. 2017;114(13):3515-20.

39. Hill DL, Wilson DW, Sampaio NG, Eriksson EM, Ryg-Cornejo V, Harrison GLA, et al. Merozoite antigens of Plasmodium falciparum elicit straintranscending opsonizing immunity. Infect Immun. 2016;84(8):2175-84. https://doi.org/10.1128/IAl.00145-16.

40. Nguyen TN, von Seidlein L, Nguyen TV, Truong PN, Hung SD, Pham HT, et al. The persistence and oscillations of submicroscopic Plasmodium falciparum and Plasmodium vivax infections over time in Vietnam: an open cohort study. Lancet Infect Dis. 2018;18(5):565-72. https://doi.org/10.1016/ S1473-3099(18)30046-X.

41. Fowkes FJ, Richards JS, Simpson JA, Beeson JG. The relationship between anti-merozoite antibodies and incidence of Plasmodium falciparum malaria: a systematic review and meta-analysis. PLoS Med. 2010;7(1):e1000218. https://doi.org/10.1371/journal.pmed.1000218.

42. McLean ARD, Wai HP, Thu AM, Khant ZS, Indrasuta C, Ashley EA, et al. Malaria elimination in remote communities requires integration of malaria control activities into general health care: an observational study and interrupted time series analysis in Myanmar. BMC Med. 2018;16(1):183 https://doi.org/10.1186/s12916-018-1172-x.

43. Srisutham S, Suwannasin K, Mathema VB, Sriprawat K, Smithuis FM, Nosten $F$, et al. Utility of Plasmodium falciparum DNA from rapid diagnostic test kits for molecular analysis and whole genome amplification. Malar J. 2020;19(1): 193. https://doi.org/10.1186/s12936-020-03259-9.

44. Robinson A, Busula AO, Muwanguzi JK, Powers SJ, Masiga DK, Bousema T, et al. Molecular quantification of Plasmodium parasite density from the 
blood retained in used RDTs. Scientific Reports. 2019;9(1):5107. https://doi. org/10.1038/s41598-019-41438-0.

45. Williams GS, Mweya C, Stewart L, Mtove G, Reyburn H, Cook J, et al. Immunophoretic rapid diagnostic tests as a source of immunoglobulins for estimating malaria sero-prevalence and transmission intensity. Malar J. 2009; 8(1):168. https://doi.org/10.1186/1475-2875-8-168.

\section{Publisher's Note}

Springer Nature remains neutral with regard to jurisdictional claims in published maps and institutional affiliations.

\section{Ready to submit your research? Choose BMC and benefit from:}

- fast, convenient online submission

- thorough peer review by experienced researchers in your field

- rapid publication on acceptance

- support for research data, including large and complex data types

- gold Open Access which fosters wider collaboration and increased citations

- maximum visibility for your research: over $100 \mathrm{M}$ website views per year

At $\mathrm{BMC}$, research is always in progress. 